\title{
FEATURE SELECTION APPROACH IN ANIMAL CLASSIFICATION
}

\author{
Y H Sharath Kumar and C D Divya \\ Department of Computer Science and Engineering, \\ Maharaja Institute of Technology, Mysore \\ Belawadi, Srirangapatna Tq, Mandya - 571438 \\ Karanataka India
}

\begin{abstract}
In this paper, we propose a model for automatic classification of Animals using different classifiers Nearest Neighbour, Probabilistic Neural Network and Symbolic. Animal images are segmented using maximal region merging segmentation. The Gabor features are extracted from segmented animal images. Discriminative texture features are then selected using the different feature selection algorithm like Sequential Forward Selection, Sequential Floating Forward Selection, Sequential Backward Selection and Sequential Floating Backward Selection. To corroborate the efficacy of the proposed method, an experiment was conducted on our own data set of 25 classes of animals, containing 2500 samples. The data set has different animal species with similar appearance (small inter-class variations) across different classes and varying appearance (large intra-class variations) within a class. In addition, the images of flowers are of different poses, with cluttered background under different lighting and climatic conditions. Experiment results reveal that Symbolic classifier outperforms Nearest Neighbour and Probabilistic Neural Network classifiers.
\end{abstract}

\section{KEYWORDS}

Region Merging, Feature Selection, Gabor Filters, Nearest Neighbour, Probabilistic Neural Network, Symbolic classifier

\section{INTRODUCTION}

Advances in hardware and corresponding drop in prices of digital cameras have increased the availability of digital photographs of wild animal sightings at high resolutions and qualities, making fully-automatic or computer assisted animal identification is an attractive approach. These flaws can be curbed by applying computer vision algorithms for developing an automated animal detection and classification system. Animal classification is the most important in monitoring animal locomotive behaviour and its interaction with the environment. The new zoological systems like radiofrequency identification (RFID) and global positioning system (GPS) is developed for animal trace facility, identification, anti-theft, security of animals in zoo.

Developing system for classifying of animals is very challenging task. The photographs captured from low quality camera sensors e.g. low frame rate, low resolution, low bit depth and colour distortion. Additionally, the animal appearance caused by several complicated factors like viewpoint, scale, illumination, partial occlusions, multiple instances. Lastly, the greatest challenge lies in preserving the intra-class and inter-class variability's. 
Signal \& Image Processing : An International Journal (SIPIJ) Vol.5, No.4, August 2014

\section{RELATED WORK}

In this section, we provide a brief review on existing work related to our work.

Animal classification has majorly four stages in it, viz., segmentation, feature extraction, tracking and classification.

Before extraction of features from an animal image or frame, the region corresponding to animal has to be segmented from its background. Given an image with animal, the goal is to segment out the animal region which is the only region of our interest in it. In the second step, different features are chosen to describe different properties of the animal. Some animals are with very high distinctive shapes, some have distinctive colour, some have distinctive texture patterns, and some are characterized by a combination of these properties. Finally extracted features are used to classify the animal. Segmentation subdivides an image into its constituent parts or object. Segmentation process should stop when the object of interest has been isolated. Animals are often surrounded by greenery, shadow in the background due to which the regions corresponding to the animal in the scene and the background may look very similar.

Pixel based segmentation uses only pixel appearance to assign a label to a pixel (Das et al., [1], Tobias et al., [2]). Region based segmentation is another type of segmentation. The main goal of this segmentation is to partition an image into regions by looking for the boundaries between region based on discontinuities in gray level or colour properties. These include (Mukhopadhyay and Chanda, [3]). Graph-based labeling methods, where a global energy function is defined depending on both appearance and image gradients. These include (Boykov and Jolly, [4], Nilsback and Zisserman, [5]).

Tilo and Janko [6] proposed a method for animal classification using facial features. Lahiri et al., [7] used the coat marking to differentiate the individual zebra. Ardovini et al., [8] presented a model for identification of elephants based on the shape of the nicks characterizing the elephant ears. Deva et al., [9] proposed 2D articulated models known as pictorial structures from videos of animals. This model can be used to detect the animal in the videos by using the texture feature of the animals. The animals are classified using unsupervised learning (Pooya et al., [10]). Heydar et al., [11] developed an animal classification system using joint textural information. Human/animal classification for unattended ground sensors uses wavelet statistics based on average, variance and energy of the third scale residue and spectral statistics based on amplitude and shape features for robust discrimination [12]. Matthias [13] focused on automated detection of elephants in wildlife video which uses color models. Xiao et al., [14] proposed a method for automated identification of animal species in camera trap images. In this method they used dense SIFT descriptor and cell-structured LBP (cLBP).

After feature extraction, the challenge lies in deciding suitable classifier. Mansi et al., [15] proposed a model for Animal detection using template matching. Matthias [13] used colour features for animal classification using Support Vector Machine (SVM) classifier. Deva et al., [9] tried a variety of classifiers, such as K-way logistic regression, SVM, and K-Nearest Neighbours]. Tree based classifiers are capable of modelling complex decision surfaces [12]. Heydar et al., [11] use three different classifiers like Single Histogram, SVM and Joint Probability model. Pooya et al., [10] present a model for animal detect by comparing with ground truth created by human experts. Xiaoyuan et al., [14] used linear SVM classifier for the classification of animals.

In this work, we propose to automate the task of Animal classification. We investigate the suitability of various feature selection method for effective classification. The organization of the 
Signal \& Image Processing : An International Journal (SIPIJ) Vol.5, No.4, August 2014

paper is as follows. In section 3 the proposed method is explained with a neat block diagram along with a brief introduction to Gabor texture analysis and Feature Selection methods. The experimental results using different classifiers are discussed in section 4 and the paper is concluded in section 5 .

\section{PROPOSED METHOD}

The proposed model consists of four stages - segmentation, feature extraction, feature selection and classification. The region merging segmentation is used to segment the Animal region from background. Gabor features are extracted from segmented animal image. Discriminative features are then selected using Sequential Forward Selection (SFS), Sequential Floating Forward Selection (SFFS), Sequential Backward Selection (SBS) and Sequential Floating Backward Selection (SFBS). These features are queried to Nearest Neighbour classifier, Probabilistic Neural Network classifier and Symbolic classifier to label an unknown Animal.

\subsection{Animal Segmentation}

The goal is to automatically segment out the animal present in a cluttered background. In our method, an initial segmentation is required to partition the image into homogeneous regions (figure (2b)) for merging. For initial segmentation we used Quick shift segmentation.

\subsubsection{Region Merging}

In animal segmentation, the central region of image is called as object marker region and the boundary of the image is called as background marker region. We use green markers to mark the object while using blue markers to represent the background shown in figure (2c). After object marking, each region will be labelled as one of three kinds of regions: the marker object region, the marker background region and the non-marker region. To extract the object contour, nonmarker region must be assigned to either object region or background region. The region merging method starts from the initial marker regions and all the non-marker regions will be gradually labelled as either object region or background region.

After initial segmentation, many small regions are available. The colour histograms are used as descriptors to represent the regions as the initially segmented small regions of the desired object often vary a lot in size and shape, while the colours of different regions from the same object will have high similarity. The RGB colour space is used to compute the colour histogram. Each colour channel is quantized into 16 levels and then the histogram of each region is calculated in the feature space of $16 \times 16 \times 16=4096$ bins. The key issue in region merging is how to determine the similarity between the unmarked regions with the marked regions so that the similar regions can be merged with some logic control. The Bhattacharyya coefficient is used to measure the similarity $\rho(R, Q)$ between two regions $\mathrm{R}$ and $\mathrm{Q}$, which is given by,

$$
\rho(R, Q)=\sum_{u=1}^{4096} \sqrt{\text { Hist }_{R}^{u} \cdot \text { Hist }_{Q}^{u}}
$$

where Hist $_{R}$ and Hist $_{Q}$ are the normalized histograms of $R$ and $Q$, respectively, and the superscript $u$ represents the $u^{\text {th }}$ element of them. Bhattacharyya coefficient $\rho$ is a divergencetype measure which has a straightforward geometric interpretation. It is the cosine of the angle between the unit vectors 
Signal \& Image Processing : An International Journal (SIPIJ) Vol.5, No.4, August 2014

$$
\left(\sqrt{\text { Hist }_{R}^{1}},,,,,, \sqrt{\text { Hist }_{R}^{4096}}\right)^{T} \text { and }\left(\sqrt{\text { Hist }_{Q}^{1}},,,,,,, \sqrt{\text { Hist }_{Q}^{4096}}\right)^{T}
$$

Higher the Bhattacharyya coefficient between $R$ and $Q$, higher is the similarity between them

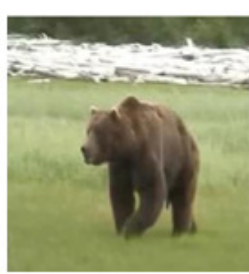

(a)

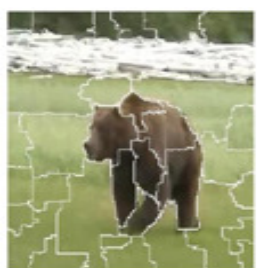

(b)

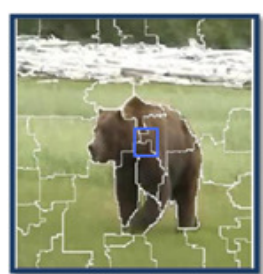

(c)

Figure 2: (a) Input image (b) Initial segmentation (c) Object regions marked
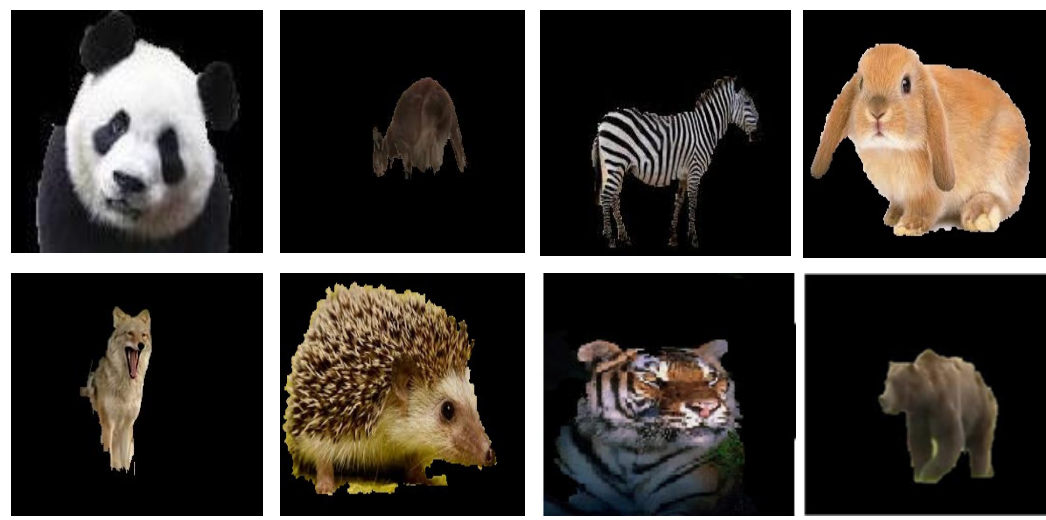

Figure 3: shows the results of animal segmentation on animal images.

\subsection{Gabor Filter Responses}

Texture analysis using filters based on Gabor functions falls into the category of frequency-based approaches. These approaches are based on the premise that the texture is an image pattern containing a repetitive structure that can be effectively characterized in a frequency domain, specifically in Fourier domain. One of the challenges, however, of such an approach is dealing with the trade-off between the joint uncertainty in the space and frequency domains. Meaningful frequency based analysis cannot be localized without bound. An attractive mathematical property of Gabor functions is that they minimize the joint uncertainty in space and frequency. They achieve the optimal trade-off between localizing the analysis in the spatial and frequency domains. Using Gabor filters to analyze texture appeals from a psycho-visual perspective as well. The texture analysis is accomplished by applying a bank of scale and orientation selective Gabor filters to an image (Newsam and Kamath [16]). These filters are constructed as follows. A twodimensional Gabor function $\mathrm{g}(\mathrm{x} ; \mathrm{y})$ and its Fourier transform $\mathrm{G}(\mathrm{u}$; v) can be written as:

$$
g(x, y)=\left(\frac{1}{2 \pi \sigma_{x} \sigma_{y}}\right) \exp \left[-\frac{1}{2}\left(\frac{x^{2}}{\sigma_{x}^{2}}+\frac{y^{2}}{\sigma_{y}^{2}}\right)+2 \pi i W x\right]
$$

and

$$
G(u, v)=\exp \left\{-\frac{1}{2}\left[\frac{(u-W)^{2}}{\sigma_{u}{ }^{2}}+\frac{v^{2}}{\sigma_{v}{ }^{2}}\right]\right\}
$$


Signal \& Image Processing : An International Journal (SIPIJ) Vol.5, No.4, August 2014

where $i=\sqrt{-1}, \sigma_{u}=1 / 2 \pi \sigma_{x}$ and $\sigma_{v}=1 / 2 \pi \sigma_{y}$ control the tradeoff between spatial and frequency resolution, and $\mathrm{W}$ controls the modulation. A class of self-similar functions referred to as Gabor wavelets is now considered. Let $\mathrm{g}(\mathrm{x}, \mathrm{y})$ be the mother wavelet. A filter dictionary can be obtained by appropriate dilations and translations of $\mathrm{g}(\mathrm{x}, \mathrm{y})$ through the generating function.

$$
\begin{aligned}
& \qquad g_{r s}(x, y)=a^{-s} g\left(x^{\prime}, y^{\prime}\right), a>1, s \in 0, \ldots \ldots . S-1, r \in 1, \ldots . . R \\
& \text { where } x^{\prime}=a^{-s}(x \cos \theta+y \sin \theta) \quad \text { and } y^{\prime}=a^{-s}(-x \sin \theta+y \cos \theta) \text { with } \theta=(r-1) \pi / R .
\end{aligned}
$$

The indices $\mathrm{r}$ and $\mathrm{s}$ indicate the orientation and scale of the filter respectively. $\mathrm{R}$ is the total number of orientations and $\mathrm{S}$ is the total number of scales in the filter bank. While the size of the filter bank is application dependent, it shall be noticed later in experimentation that a bank of filters tuned to combinations of $0,2,4,6,8$ and 10 scales, and different orientations, at 22.5 degree intervals are sufficient for flower analysis.

\subsection{Feature Selection}

Our goal is to reduce the dimension of the data by finding a small set of important features which can give good classification performance. Feature selection algorithms can be roughly grouped into two categories: filter methods and wrapper methods. Filter methods rely on general characteristics of the data to evaluate and to select the feature subsets without involving the chosen learning algorithm. Filters are usually used as a pre-processing step since they are simple and fast. Wrapper methods use the performance of the chosen learning algorithm to evaluate each candidate feature subset. Wrapper methods search for features better fit for the chosen learning algorithm, but they can be significantly slower than filter methods if the learning algorithm takes a long time to run.

Large numbers of diverse acoustic hi-level features were discussed considering their performance. However, sparse analysis of single feature relevance by means of filter or wrapper based evaluation has been fulfilled, yet. Features are mostly reduced by means of the well known Principal Component Analysis and selection of the obtained artificial features corresponding to the highest Eigen-values As such reduction still requires calculation of the original features we aim at a real elimination of original features within the set. As search function within feature selection (FS) we apply different feature selection algorithms [17] like SFS (Sequential Feature Selection), SBS (Sequential Backward Selection), SFFS (Sequential Floating Forward Selection) and SFBS (Sequential Floating Backward Selection,which is well known methods for high performance. Thereby the evaluation function is the classifier, in our case Nearest Neighbor, Probabilistic Neural Network and Symbolic Classifier is used. This optimizes the features as a set rather than finding single features of high performance. The search is performed by forward and backward steps eliminating and adding features in a floating manner to an initially empty set.

The feature selection algorithm is performed for methods like Cross Validation (Standard Cross Validation (SCV), A-Cross Validation (ACV), AB-Cross Validation (ABCV) and Resubstitution. Sequential feature selection is one of the most widely used techniques. It selects a subset of features by sequentially adding (forward search-SFS) or removing (backward search-SBS) until stopping conditions are satisfied. There are two main categories of floating search methods: forward (SFFS) and backward (SFBS). Basically in the case of forward search (SFFS), the algorithm starts with a null feature set and for each step the best feature that satisfies criterion function is included with the current feature set. In the case of worst feature (concerning the criterion) is eliminated from the set, it is performed one step of sequential backward selection (SBS). Therefore, the SFFS proceeds dynamically increasing and decreasing the number of features until the desired condition is reached. 
Signal \& Image Processing : An International Journal (SIPIJ) Vol.5, No.4, August 2014

\subsection{Classification}

The extracted features are fed to fusion of classifiers such as nearest neighbor, Probabilistic Neural Network and symbolic classifiers. Introductions on each of these classifiers are given in the flowing subsections.

\subsubsection{Nearest Neighbour (NN)}

One of the simplest classifiers which we used is the Nearest Neighbor classifier (Bremner [18], Hall [19]). The term nearest can be taken to mean the smallest Euclidean distances in ndimensional feature space. This takes a test sample feature in a vector form, and finds the Euclidean distance between this and the vector representation of each training example. The training sample closest to the test sample is termed as its Nearest Neighbor. Since the trained sample in some sense is the one most similar to our test sample, it makes sense to allocate its class label to the test sample. This exploits the 'smoothness' assumption that samples near each other are likely to have the same class.

\subsubsection{Probabilistic Neural Networks (PNN)}

Probabilistic neural networks (Specht [20][21]) are feed forward networks built with three layers. They are derived from Bayes Decision Networks. They train quickly since the training is done in one pass of each training vector, rather than several. Probabilistic neural networks estimate the probability density function for each class based on the training samples. The probabilistic neural network uses a similar probability density function. This is calculated for each test vector. Vectors must be normalized prior to their input into the network. There is an input unit for each dimension in the vector. The input layer is fully connected to the hidden layer. The hidden layer has a node for each classification. The output layer has a node for each pattern classification. The probabilistic neural network trains immediately but execution time is slow and it requires a large amount of space in memory.

\subsubsection{Symbolic Representation}

In this section, we use extracted Gabor features for symbolic representation of animal samples. As features of animal samples have considerable intra class variations in each subgroup, using conventional data representation, preserving these variations is difficult. Hence, the proposed work is intend to use unconventional data processing called symbolic data analysis which has the ability to preserve the variations among the data more effectively. In this work, symbolic representation (Guru and Prakash, [22]) has been adapted to capture these variations through feature assimilation by the use of an interval valued feature vector as follows.

Let $\left[\mathrm{T}_{1}, \mathrm{~T}_{2}, \mathrm{~T}_{3}, \ldots, \mathrm{T}_{\mathrm{n}}\right]$ be a set of $n_{t}$ samples of a animal class say $C_{j} ; j=1,2,3, \ldots N$ (N denotes number of classes) and let $F_{i}=\left[d_{i 1}, d_{i 2}, d_{i 3}, d_{i 4}, \ldots, d_{i m}\right]$ be the set of $\mathrm{m}$ features characterizing the animal sample $T_{i}$ of the class $C_{j}$. Let $\operatorname{Min}_{\mathrm{jk}}$ be the minimum of the $k^{\text {th }}$ feature values obtained from all the n samples of the class $C_{j}$ and let $\operatorname{Max}_{\mathrm{jk}}$ be the maximum of the feature values obtained from all the $\mathrm{n}$ samples of the class $C_{j}$.

$$
\operatorname{Min}_{j k}=\min \left(d_{i k}\right) \quad \operatorname{Max}_{j k}=\max \left(d_{i k}\right) \quad \text { where } \mathrm{k}=1 \text { to } \mathrm{n}
$$

Now, we recommend to capture the intra class variations in each $k^{\text {th }}$ feature value of the $\mathrm{j}^{\text {th }}$ class in form of interval valued feature. That is, each class $C_{j}$ is represented by the use of interval valued features 
Signal \& Image Processing : An International Journal (SIPIJ) Vol.5, No.4, August 2014

$$
\left(\left[\mathrm{d}_{\mathrm{j} 1}^{-}, d_{\mathrm{j} 1}^{+}\right],\left[\mathrm{d}_{\mathrm{j} 2}^{-}, d_{\mathrm{j} 2}^{+}\right], \ldots .,\left[\mathrm{d}_{\mathrm{jk}}^{-}, d_{\mathrm{jk}}^{+}\right]\right) \text {where } \mathrm{d}_{\mathrm{jk}}^{-}=\operatorname{Min}_{\mathrm{jk}} \text { and } d_{\mathrm{jk}}^{+}=\operatorname{Max}_{\mathrm{jk}}
$$

Each interval representation depends on the minimum and maximum of respective individual

features. The interval $\left[\mathrm{d}_{\mathrm{jk}}^{-}, d_{\mathrm{jk}}^{+}\right]$represents the upper and lower limits of the $k^{\text {th }}$ feature values of the class $C_{j}$. Now, a reference animal representing the entire class (all the samples of animal) is formed by use interval type and is given by

$$
R F_{j}=\left\{\left[d_{j 1}^{-}, d_{j 1}^{+}\right],\left[d_{j 2}^{-}, d_{j 2}^{+}\right], \ldots,\left[d_{j m}^{-}, d_{j m}^{+}\right]\right\}
$$

where $c=1,2, \ldots$, of $\mathrm{N}$. It shall be noted that unlike conventional feature vector, this is a vector of interval-valued features and this symbolic feature vector is stored in the knowledgebase as a representative of the $\mathrm{j}^{\text {th }}$ class. We recommend computing symbolic feature vectors for each individual sample of a class and store them in the knowledgebase for future recognition requirements. Thus, the knowledgebase has $N$ number of symbolic vectors.

\subsubsection{Classification}

In this section we use the symbolic classifier (Guru and Prakash, [22]) for classifying the animals. In classification model, a test sample of an unknown animal is described by a set of $m$ features values of crisp type and compares it with the corresponding interval type features of the respective symbolic reference samples $R_{j}$, stored in the knowledgebase to ascertain the efficiency.

Let $F_{i}=\left[d_{t 1}, d_{t 2}, d_{t 3}, d_{t 4}, . ., d_{t m}\right]$ be an $m$ dimensional vector describing a test sample. Let $R F_{c}$; $c=1,2,3, \ldots, N$ be the representative symbolic feature vectors stored in knowledgebase. During animal classification process each $k^{\text {th }}$ feature value of the test animal sample is compared with the respective intervals of all the representatives to examine if the feature value of the test animal sample lies within them. The test animal sample is said to belong to the class with which it has a maximum acceptance count $A_{c}$.

Acceptance count $\mathrm{A}_{\mathrm{C}}$ is given by,

$$
\mathrm{A}_{\mathrm{C}}=\sum_{\mathrm{k}=1}^{m} \mathrm{C}\left(\mathrm{d}_{\mathrm{tk}},\left[\mathrm{d}_{\mathrm{tk}}^{-}, d_{\mathrm{jk}}^{+}\right]\right)
$$

where,

$$
\mathrm{C}\left(\mathrm{d}_{\mathrm{tk}},\left[\mathrm{d}_{\mathrm{tk}}^{-}, d_{\mathrm{jk}}^{+}\right]\right)= \begin{cases}1 & \text { if }\left(\mathrm{d}_{\mathrm{tk}} \geq d_{\mathrm{jk}}^{-} \text {and } d_{\mathrm{tk}} \leq d_{\mathrm{jk}}^{+}\right) \\ 0 & \text { otherwise }\end{cases}
$$

When the database happens to be large, there is a possibility for a test animal sample to possess the same maximum acceptance count with two or more animal classes. Under such circumstances we recommend to resolve the conflict by the use of the following similarity measure (Guru and Prakash, [22]) which computes the similarity value between a test animal sample and each of the conflicting classes say $\mathrm{j}^{\text {th }}$ class.

$$
\text { Total_Sim }\left(\mathrm{F}_{\mathrm{t}}, \mathrm{RF}_{\mathrm{j}}\right)=\sum_{\mathrm{k}=1}^{m} \mathrm{C}\left(\mathrm{d}_{\mathrm{tk}},\left[\mathrm{d}_{\mathrm{tk}}^{-}, d_{\mathrm{jk}}^{+}\right]\right)
$$

Here $\left[\mathrm{d}_{\mathrm{jk}}^{-}, d_{\mathrm{jk}}^{+}\right]$represents the $\mathrm{k}^{\text {th }}$ feature interval of the $\mathrm{j}^{\text {th }}$ conflicting class, and 
Signal \& Image Processing : An International Journal (SIPIJ) Vol.5, No.4, August 2014

$$
\mathrm{C}\left(\mathrm{d}_{\mathrm{t} k},\left[\mathrm{~d}_{\mathrm{jk}}, d_{\mathrm{jk}}^{+}\right]\right)=\left\{\begin{array}{l}
1 \\
\max \left(\frac{1}{1+d_{\mathrm{tk}}-\mathrm{d}_{\mathrm{jk}} \mid * \delta}, \frac{1}{1+d_{\mathrm{tk}}-d_{\mathrm{jk}}^{+} \mid * \delta}\right)+1 \text { otherwise }
\end{array}\right.
$$

where $\delta$ is a normalizing factor.

\section{DATASETS}

In this work we have created our own database despite of existence of other databases as these are less intra class variations or no change in view point. We collected Animal images from World Wide Web in addition to taking up some photographs of Animals that can be found in and around our place. The images are taken to study the effect of the proposed method with large inter, intra class variations and lighting conditions. The dataset consists of 25 classes of Animal with 100 images of each, totally 2500 . Figure 4 shows a sample image of each 25 classes

\section{EXPERIMENTATION}

In this experimentation we intend to study the accuracy of different feature selection algorithms. The experimentation has been conducted on database of 25 classes under varying number of training samples 40, 60 and 80 from each class. Table 1 shows the classification accuracy of animals obtained by different classifiers like KNN, PNN and Symbolic classifiers without feature selection under varying training samples. Additional, we tabulate the time taken by individual classifiers. Table 2 to Table 5 shows the classification accuracy of animals obtained by different classifiers like KNN, PNN and Symbolic classifiers for feature selection algorithms using different methods like Standard Cross Validation, A- Cross Validation, AB- Cross Validation, Resubstitution. From tables we can observe that symbolic classifier achievers maximum accuracy of 92.5 without feature selection and 87 with AB-Cross validation (SBS). Respectively The KNN classifier achieves maximum accuracy of 84.4 with A-Cross validation(SFBS)and PNN classifier achieves maximum accuracy of 83.8 with Standard cross validation(SFS).
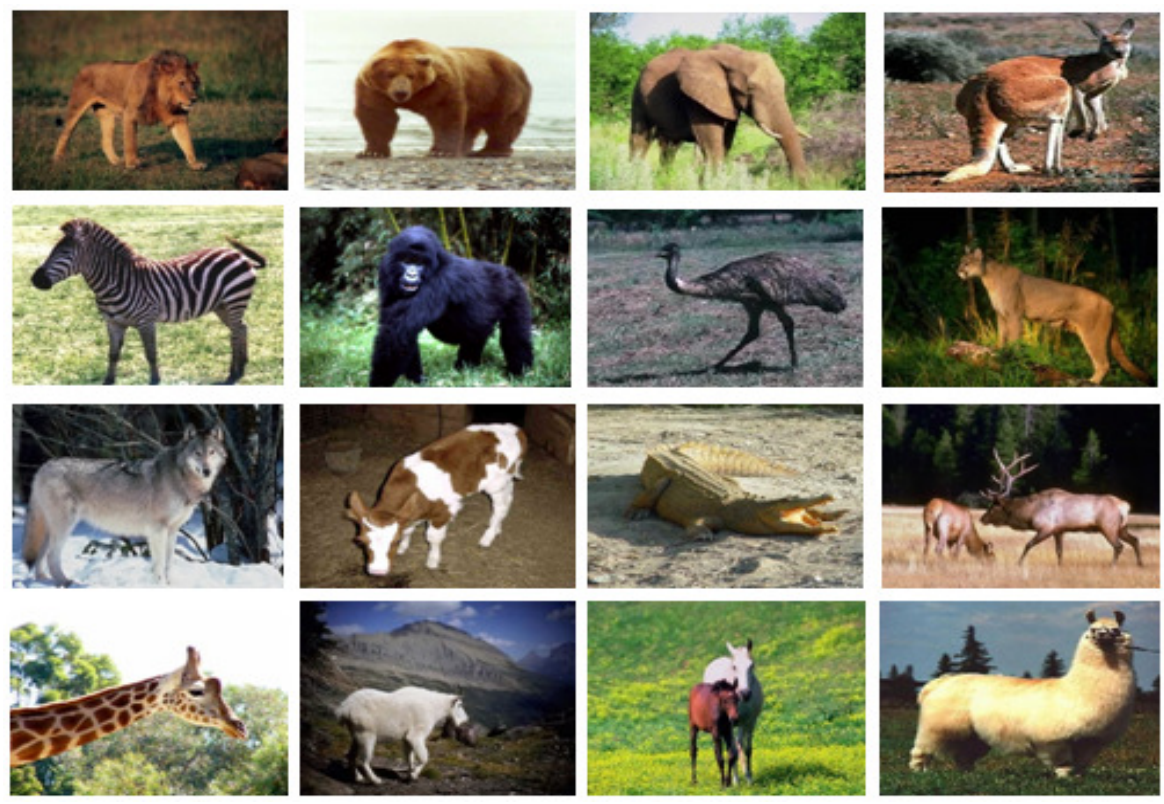

Figure 4: Sample Animal images of 25 flower classes considered in this work 
Signal \& Image Processing : An International Journal (SIPIJ) Vol.5, No.4, August 2014

Table 1: Shows Accuracy for different classifiers without using Feature selection

\begin{tabular}{|c|c|c|c|c|c|c|}
\hline \multirow{2}{*}{ Classifiers } & \multicolumn{7}{|c|}{ Percentage of Training } \\
\cline { 2 - 7 } & \multicolumn{2}{|c|}{80} & \multicolumn{2}{|c|}{60} & \multicolumn{2}{c|}{40} \\
\cline { 2 - 7 } & Result & $\begin{array}{c}\text { Time in } \\
\text { sec }\end{array}$ & Result & $\begin{array}{c}\text { Time in } \\
\text { sec }\end{array}$ & Result & $\begin{array}{c}\text { Time in } \\
\text { sec }\end{array}$ \\
\hline Symbolic & 92.5 & 0.09 & 90.5 & 0.12 & 90.4 & 0.16 \\
\hline KNN & 84.5 & 6.02 & 82 & 6.65 & 77.66 & 6.98 \\
\hline PNN & 83.6 & 1.52 & 80.3 & 1.82 & 76.6 & 1.98 \\
\hline
\end{tabular}

Table 2: Shows Accuracy for different classifiers using Standard Cross Validation

\begin{tabular}{|c|c|c|c|c|c|c|}
\hline \multicolumn{7}{|c|}{ CCR Estimation Method : Standard Cross Validation(SCV) } \\
\hline \multirow[t]{2}{*}{ Feature Selection Methods } & \multicolumn{6}{|c|}{ Percentage of Training } \\
\hline & \multicolumn{2}{|c|}{80} & \multicolumn{2}{|c|}{60} & \multicolumn{2}{|c|}{40} \\
\hline \multicolumn{7}{|c|}{ Symbolic Classifier } \\
\hline & Result & $\begin{array}{c}\text { Time in } \\
\text { sec }\end{array}$ & Result & $\begin{array}{c}\text { Time in } \\
\text { sec }\end{array}$ & Result & $\begin{array}{c}\text { Time in } \\
\text { sec }\end{array}$ \\
\hline SFS & 84.1 & 0.24 & 84.2 & 0.45 & 82.2 & 0.45 \\
\hline SFFS & 84.4 & 0.52 & 83.4 & 0.81 & 84 & 1.16 \\
\hline SFBS & 84.2 & 1.44 & 84.1 & 1.52 & 82 & 1.54 \\
\hline SBS & 84.3 & 0.64 & 83.7 & 1.05 & 84 & 1.51 \\
\hline \multicolumn{7}{|c|}{ KNN Classifier } \\
\hline SFS & 80.8 & 5.35 & 79.1 & 6.23 & 76.8 & 6.25 \\
\hline SFFS & 81.1 & 5.28 & 80.2 & 5.87 & 78.2 & 6.30 \\
\hline SFBS & 80.1 & 6.12 & 78.4 & 5.32 & 78.1 & 5.14 \\
\hline SBS & 83 & 5.23 & 78.4 & 5.84 & 77.7 & 5.91 \\
\hline \multicolumn{7}{|c|}{ PNN Classifier } \\
\hline SFS & 83.8 & 1.24 & 81.8 & 1.25 & 78.4 & 1.31 \\
\hline SFFS & 82.4 & 1.13 & 78 & 2.82 & 75.2 & 2.95 \\
\hline SFBS & 78.2 & 2.45 & 75 & 2.34 & 74.1 & 2.61 \\
\hline SBS & 80.8 & 2.18 & 79.4 & 2.54 & 75.2 & 2.36 \\
\hline
\end{tabular}


Signal \& Image Processing : An International Journal (SIPIJ) Vol.5, No.4, August 2014

Table 3: Shows Accuracy for different classifiers using A- Cross Validation

\begin{tabular}{|c|c|c|c|c|c|c|}
\hline \multicolumn{7}{|c|}{ CCR Estimation Method : A Cross Validation(A-CV) } \\
\hline \multirow[t]{2}{*}{ Feature Selection Methods } & \multicolumn{6}{|c|}{ Percentage of Training } \\
\hline & \multicolumn{2}{|c|}{80} & \multicolumn{2}{|c|}{60} & \multicolumn{2}{|c|}{40} \\
\hline \multicolumn{7}{|c|}{ Symbolic Classifier } \\
\hline & Result & $\begin{array}{l}\text { Time in } \\
\text { sec }\end{array}$ & Result & $\begin{array}{l}\text { Time in } \\
\text { sec }\end{array}$ & Result & $\begin{array}{c}\text { Time in } \\
\text { sec }\end{array}$ \\
\hline SFS & 81.6 & 0.26 & 79.2 & 0.51 & 80.1 & 0.72 \\
\hline SFFS & 84.3 & 0.49 & 84.2 & 0.91 & 83.6 & 1.27 \\
\hline SFBS & 88.5 & 0.77 & 87.1 & 1.24 & 85.3 & 1.78 \\
\hline SBS & 84.8 & 0.63 & 78.2 & 0.146 & 76.2 & 1.14 \\
\hline \multicolumn{7}{|c|}{ KNN Classifier } \\
\hline SFS & 75.4 & 6.25 & 74.4 & 6.88 & 71.6 & 6.78 \\
\hline SFFS & 82.2 & 6.95 & 79.6 & 7.25 & 76.7 & 7.27 \\
\hline SFBS & 84.4 & 4.21 & 80.2 & 5.08 & 78.4 & 5.85 \\
\hline SBS & 84.5 & 6.82 & 80.8 & 6.94 & 79.1 & 6.74 \\
\hline \multicolumn{7}{|c|}{ PNN Classifier } \\
\hline SFS & 75.2 & 1.92 & 74.2 & 2.45 & 72.1 & 2.32 \\
\hline SFFS & 77.6 & 2.85 & 75.3 & 2.92 & 73.4 & 2.95 \\
\hline SFBS & 83.2 & 2.98 & 78.8 & 3.12 & 75.8 & 3.36 \\
\hline SBS & 78.6 & 2.90 & 76.5 & 2.36 & 73.4 & 2.98 \\
\hline
\end{tabular}

Table 4: Shows Accuracy for different classifiers using AB- Cross Validation

\begin{tabular}{|c|c|c|c|c|c|c|}
\hline \multicolumn{7}{|c|}{ CCR Estimation Method : AB Cross Validation(AB-CV) } \\
\hline \multirow[t]{2}{*}{ Feature Selection Methods } & \multicolumn{6}{|c|}{ Percentage of Training } \\
\hline & \multicolumn{2}{|c|}{80} & \multicolumn{2}{|c|}{60} & \multicolumn{2}{|c|}{40} \\
\hline \multicolumn{7}{|c|}{ Symbolic Classifier } \\
\hline & Result & $\begin{array}{l}\text { Time in } \\
\text { seconds }\end{array}$ & Result & $\begin{array}{l}\text { Time in } \\
\text { seconds }\end{array}$ & Result & $\begin{array}{l}\text { Time in } \\
\text { seconds }\end{array}$ \\
\hline SFS & 80.9 & 0.23 & 80.7 & 0.42 & 80.2 & 0.67 \\
\hline SFFS & 82.2 & 0.25 & 82 & 0.43 & 80.2 & 0.68 \\
\hline SFBS & 84.9 & 0.66 & 83 & 1.12 & 82.1 & 1.51 \\
\hline SBS & 87 & 0.64 & 86.4 & 1.15 & 86.1 & 1.53 \\
\hline \multicolumn{7}{|c|}{ KNN Classifier } \\
\hline SFS & 77.6 & 5.23 & 75.4 & 5.48 & 73.8 & 6.21 \\
\hline SFFS & 77.2 & 5.36 & 72.3 & 5.56 & 71.4 & 5.92 \\
\hline SFBS & 84.2 & 6.32 & 80.7 & 6.45 & 78.2 & 6.58 \\
\hline SBS & 83.2 & 5.98 & 77.8 & 5.63 & 76.6 & 6.03 \\
\hline \multicolumn{7}{|c|}{ PNN Classifier } \\
\hline SFS & 80.8 & 1.67 & 78.9 & 1.78 & 75.6 & 2.03 \\
\hline SFFS & 77.4 & 1.98 & 74.3 & 2.05 & 72.8 & 2.67 \\
\hline SFBS & 81.2 & 2.35 & 78.1 & 2.89 & 74.5 & 2.96 \\
\hline SBS & 78.4 & 2.08 & 76.1 & 2.97 & 75.2 & 2.98 \\
\hline
\end{tabular}


Signal \& Image Processing : An International Journal (SIPIJ) Vol.5, No.4, August 2014

Table 5: Shows Accuracy for different classifiers using Resubstitution

\begin{tabular}{|c|c|c|c|c|c|c|}
\hline \multicolumn{7}{|c|}{ CCR Estimation Method : Resubstitution } \\
\hline \multirow[t]{2}{*}{ Feature Selection Methods } & \multicolumn{6}{|c|}{ Percentage of Training } \\
\hline & \multicolumn{2}{|c|}{80} & \multicolumn{2}{|r|}{60} & \multicolumn{2}{|c|}{40} \\
\hline \multicolumn{7}{|c|}{ Symbolic Classifier } \\
\hline & Result & $\begin{array}{l}\text { Time in } \\
\text { seconds }\end{array}$ & Result & $\begin{array}{l}\text { Time in } \\
\text { seconds }\end{array}$ & Result & $\begin{array}{l}\text { Time in } \\
\text { seconds }\end{array}$ \\
\hline SFS & 87.4 & 0.19 & 85.5 & 0.28 & 85.2 & 0.37 \\
\hline SFFS & 87.8 & 0.28 & 85.8 & 0.36 & 85.1 & 0.45 \\
\hline SFBS & 85.7 & 1.02 & 85.5 & 0.09 & 83.4 & 1.99 \\
\hline SBS & 85.1 & 0.81 & 84.4 & 1.35 & 83.1 & 1.75 \\
\hline \multicolumn{7}{|c|}{ KNN Classifier } \\
\hline SFS & 81.4 & 5.82 & 80.5 & 5.89 & 77.5 & 6.85 \\
\hline SFFS & 84.4 & 6.93 & 79.7 & 6.87 & 77.6 & 6.99 \\
\hline SFBS & 80.8 & 6.52 & 80.2 & 6.98 & 77.3 & 6.58 \\
\hline SBS & 82.8 & 5.83 & 78.5 & 5.97 & 74.3 & 6.93 \\
\hline \multicolumn{7}{|c|}{ PNN Classifier } \\
\hline SFS & 78.2 & 1.95 & 76.5 & 1.98 & 76.4 & 1.76 \\
\hline SFFS & 76.5 & 1.96 & 75.8 & 2.05 & 74.6 & 2.67 \\
\hline SFBS & 78.6 & 2.03 & 75.5 & 2.76 & 73.8 & 2.83 \\
\hline SBS & 81.8 & 2.15 & 77.4 & 2.63 & 74.9 & 2.67 \\
\hline
\end{tabular}

\section{CONCLUSIONS}

In this paper we have proposed an Animal classification system using different classifiers like NN, PNN and Symbolic. The animal images are segmented using maximal region merging method. The Gabor features are extracted from segmented images. Discriminative gabor features are then selected using the different feature selection algorithm like Sequential Forward Selection, Sequential Floating Forward Selection, Sequential Backward Selection and Sequential Floating Backward Selection. For effectiveness of the proposed method, we have also created our own database of animals of 25 classes each consisting of 100 Animal images, totally 2500. To conduct the experimentation we have considered different size of database and studied the effect of classification accuracy. The experimental results have shown that the symbolic classifier outperforms the other individual features.

\section{REFERENCES}

[1] Das, M., Manmatha R., Riseman E. M., (1999), Indexing flower patent images using domain knowledge, IEEE Intelligent systems, vol. 14, pp. 24-33.

[2] Tobias, Orlando J., Seara R.,(2002), Image segmentation by histogram thresholding using fuzzy sets . IEEE Transactions on Image Processing, vol-11, pp. 1457-1465.

[3] Mukhopadhyay, S., Chanda, B., (2003), Multiscale morphological segmentation of gray-scale images, Image Processing, IEEE Transaction, vol.12, pp.533-549.

[4] Boykov, Y.Y., Jolly, M.P., (2001), Interactive graph cuts for optimal boundary and region segmentation of objects in N-D images. In: Proceedings of International Conference on Computer Vision (ICCV - 01), vol. 2, pp. 105-112.

[5] Nilsback, M. E., Zisserman, A.,(2004), Delving into the whorl of flower segmentation. In: The Proceedings of British Machine Vision Conference, vol. 1, pp. 27-30.

[6] Tilo B., Janko C., (2006), Real-time Face Detection and Tracking of Animals. IEEE 8th Seminar on Neural Network Applications in Electrical Engineering (NEUREL06), pp. 27-32. 
Signal \& Image Processing : An International Journal (SIPIJ) Vol.5, No.4, August 2014

[7] Lahiri M., Chayant T., Rosemary W., Daniel R., Tanya B W.,(2011), Biometric animal databases from field photographs: Identification of individual zebra in the wild, In: Proceedings of the 1st ACM International Conference on Multimedia Retrieval, pp 1-8.

[8] Ardovini. A., Cinque. L., Sangineto E., (2008), Identifying elephant photos by multi-curve matching. In: Journal of Pattern Recognition, vol 41, pp. 1867-1877.

[9] Deva R., David A. F., Kobus B., (2006), Building models of animals from video. In: IEEE transaction on Pattern Analysis and Machine Intelligence, vol 28, pp. 1319--1334.

[10] Pooya K., Jiangping W., and Thomas H., (2012), Multiple animal species detection using robust principal component analysis and large displacement optical flow, pp 1-4.

[11] Heydar A., Targhi H M, Eklundh A T., Pronobis J O., (2008), Joint visual vocabulary for animal classification, Pattern Recognition, ICPR 2008, 19th International Conference, pp 1- 4.

[12] Matthias Z, Angela S. S, Christian B., (2013), Acoustic detection of elephant presence in noisy environments, In: Proceedings of the 2nd ACM international workshop on Multimedia Analysis for Ecological Data, pp 3-8.

[13] Matthias Z., (2013), Automated detection of elephants in wildlife video, In: EURSIP Journal on Image and Video Processing, pp 1-23.

[14] Xiao T Y., Xiaobai L., Shuicheng Y., (2012), Visual Classification With Multitask Joint Sparse Representation, In proceedings of the IEEE Transactions on Image Processing, vol 21, pp 43494360.

[15] Mansi P., Miral P., (2013), Animal identification using template matching algorithm, In: International Journal of Research in Modern Engineering and Modern Technology, vol 1, pp. 26-32.

[16] Newsam, S. D., and Kamath, C.,(2004), Retrieval using texture features in high resolution multispectral satellite imagery, In SPIE Conference on Data Mining and Knowledge Discovery: Theory, Tools, and Technology VI.

[17] D.Ververidis and C.Kotropoulos, (2005), Emotional Speech Classification Using Gaussian Mixture Models and the Sequential Floating Forward Selection Algorithm, ICME, pp.1500-1503.

[18] Hall P, Park BU, Samworth R J., (2008), Choice of neighbor order in nearest-neighbor classification, Annals of Statistics, 36 (5): 2135-2152.

[19] Bremner D, Demaine E, Erickson J, Iacono J, Langerman S, Morin P, Toussaint G., (2005), Outputsensitive algorithms for computing nearest-neighbor decision boundaries. Discrete and Computational Geometry, 33 (4), 593-604.

[20] Specht, D.F., (1990), Probabilistic Neural Networks, Neural Networks, vol. 3, pp.109-118.

[21] Specht, D.F., (1990), Probabilistic Neural Networks and the Polynomial Adaline as Complementary Techniques for Classification, IEEE Transactions on Neural Networks, vol. 1, pp. 111-121.

[22] Guru, D.S. Prakash, H.N.,(2009), On-line signature verification and recognition: An approach based on symbolic representation, IEEE Transaction on Pattern Analysis and Machine Intelligence 31, 1059-1073.

\section{AUTHOR}

Y.H. Sharath Kumar is Assistant Professor in the Department of Computer Science and Engineering Maharaja Institute of Technology, Mysore. He obtained his Bachelor of Engineering from VTU Belgaum. He received his Masters degree in Computer Science and Technology from University of Mysore. He is pursuing doctoral degree under the guidance of D.S Guru under University of Mysore.

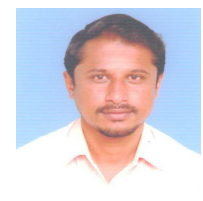

